\title{
La corrupción como estrés sobre el sistema político: cuatro escenarios que explican las estrategias de defensa del statu quo ${ }^{+}$
}

\author{
JOSEPH POZSGAI* \\ University of Tsukuba \\ jpozsgai@dailycorruption.info
}

https://doi.org/10.18800/rcpg.201702.004

\section{RESUMEN}

Actualmente, es posible considerar a la corrupción pública como el fenómeno más insidioso y peligroso para el desarrollo económico, político y social del país. La reacción tanto local como internacional a los recurrentes escándalos, sin embargo, ha sido mayormente incapaz de traducirse en la correcta implementación de políticas públicas efectivas. ¿̨or qué las demandas públicas de lucha contra la corrupción no logran romper definitivamente el statu quo? El presente artículo plantea el reconocimiento formal de incentivos ilícitos y de capital político como elementos claves que explican el nivel de voluntad política detrás de la lucha contra la corrupción. Sobre esta premisa, se propone el análisis del fenómeno de la corrupción en base a cuatro escenarios posibles en que su presencia produce estrés sobre el sistema político y amenaza la estabilidad del statu quo: (i) ineficiencia en procesos, (ii) percepción pública, (iii) intolerancia adquirida y (iv) negligencia acumulada. Por medio de la discusión de casos específicos ocurridos en las últimas tres décadas en el Perú, el artículo describe las estrategias gubernamentales que fueron adoptadas en cada ocasión para reducir demandas y estimular el apoyo popular, y que finalmente reflejaron la postura particular de los actores políticos de turno.

Palabras clave: corrupción, sistema político, estrategia gubernamental, Perú, capital político.

+ Recibido el 6 de marzo de 2017; aceptado el 3 de octubre de 2017.

* Doctor en Ciencia Política por la Universidad de Tsukuba (Japón), de la cual es asociado internacional. 


\title{
Corruption as Stress over the Political System: Four Scenarios that Explain the Defense Strategies of the Statu Quo
}

\begin{abstract}
It is currently possible to consider public corruption as the most insidious and dangerous phenomenon to economic, political, and social development in the country. Both the local and international reactions to the recurrent scandals, however, have been mostly unable to translate into the proper implementation of effective public policies. Why have the public demands to fight corruption failed to break once and for all the statu quo? This paper suggests the formal recognition of both illicit incentives and political capital as key elements to explain the level of political will to fight corruption. Building on this premise, it offers an analysis of the corruption phenomenon based on four possible scenarios though which its presence produces stress for the political system and threatens the stability of the statu quo: (i) Inefficiency in processes; (ii) public perception; (iii) acquired intolerance; and (iv) accumulated neglect. Discussing specific cases taking place over the past three decades, the paper also describes the governmental strategies that were adopted on each occasion to reduce demands and stimulate popular support, and which ultimately reflected the particular position of the incumbent actors.
\end{abstract}

Keywords: Corruption; political system; governmental strategy; Peru; political capital. 


\section{INTRODUCCIÓN}

La corrupción, como objeto de análisis, ha trascendido la esfera de las noticias internacionales y el periodismo político en la que vivió durante gran parte del siglo $\mathrm{XX}$, y puede considerarse hoy como un tema habitual de estudio en las ciencias sociales. No es posible hablar ya del fenómeno de la corrupción desde una posición relativista, siempre que su investigación actual requiere medirla con exactitud para poder incluirla como variable independiente o dependiente en un número cada vez mayor de áreas de estudio. Como resultado, el estudio de la corrupción ha crecido exponencialmente tanto en cantidad como en variedad, especialmente en lo que se refiere a las diferentes disciplinas que informan su análisis y de los elementos sometidos a escrutinio (pues la corrupción no es un concepto básico que se preste a un análisis simple y general).

Sin embargo, la actual ubicuidad de la literatura técnica y de las intervenciones gubernamentales parecen haber producido un impacto limitado: «Aunque muchos recursos se canalizan hacia la lucha contra la corrupción, hay muy pocas historias de éxito que contar cuando se trata de la implementación real de reformas anticorrupción»" (Persson, Rothstein y Teorell, 2010, p. 8).

Esta situación emerge debido, en primer lugar, al quiebre de los elementos constitutivos que componen el análisis académico en la materia (los cuales, en su mayor parte, son estudiados aisladamente y con limitada consideración a las relaciones entre ellos) y, en segundo lugar, a los supuestos implícitos en cuanto a la voluntad política de los actores encargados de adoptar las recomendaciones de política anticorrupción. Esta última característica, en particular, tiene graves consecuencias para la reducción y el control de la corrupción en entornos reales.

En cuanto a América Latina, a pesar de haber firmado la primera convención internacional anticorrupción jurídicamente vinculante hace más de veinte años (Convención Interamericana contra la Corrupción, IACAC), el Programa de las Naciones Unidas para el Desarrollo informa que «existe un alarmante bajo nivel de cumplimiento satisfactorio $»^{2}$ (UNDP, 2012, p. 46) y que «la mayoría de las recomendaciones dadas a los gobiernos no se implementan satisfactoriamente, ni se reportan adecuadamente al MESICIC, lo cual arroja dudas acerca de la verdadera voluntad y habilidad del gobierno para comprometerse con ellas» ${ }^{3}$ (UNDP, 2012, p. 45).

\footnotetext{
Traducido al espańol por el autor.

Traducido al español por el autor.

Traducido al espańol por el autor.
} 
En el caso del Perú, el Grupo de Trabajo Contra la Corrupción informó en 2010 que 43 de las 51 recomendaciones formuladas por el Mecanismo de Seguimiento de la IACAC (MESICIC) en 2004, y 21 de las 26 recomendaciones hechas en 2007, estaban aún pendientes o no había información sobre su progreso (GTCC, 2010). Como consecuencia del lento avance, solo durante 2010 y 2011, 145 personas acusadas de corrupción perpetrada en la década de los noventa fueron beneficiadas con la aplicación de la prescripción del delito.

Recientemente, la OCDE (Organización para la Cooperación y el Desarrollo Económico) ha reconocido que:

[...] el Perú tiene una legislación muy avanzada en el área de acceso a la información que data desde el 2002, pero sigue sufriendo de letargo para avanzar hacia una política más completa de [Gobierno Abierto]... y recién está dando algunos pasos iniciales para desarrollar datos abiertos en el país ${ }^{4}$ (OECD, 2014, p. 34).

Enfocándonos en áreas específicas, queda claro que, más allá de firmar acuerdos y convenciones, la élite política avanza con desidia cuando se trata de realmente implementar y hacer cumplir acciones destinadas a eventualmente reducir los niveles de corrupción en el país. En lo que respecta al control de lobbies, el Integrity Review publicado por la OCDE en 2017 señala que «a pesar de tener una regulación formal relativamente fuerte, Perú claramente tiene problemas para implementarla de manera efectiva. Hasta la fecha, solo cinco personas naturales y una persona jurídica se han registrado como lobistas siguiendo la Ley» ${ }^{5}$ (OECD, 2017, p. 151). Y en cuanto a la financiación de los partidos políticos, el mismo informe señala el vínculo entre los niveles de aprobación popular y las decisiones tomadas con respecto a la aplicación efectiva de las leyes existentes (OECD, 2017, p. 135).

Por lo tanto, el objetivo del presente estudio es abordar los desafíos políticos inherentes a la reforma anticorrupción en el Perú (y en aquellos países que enfrentan problemas similares). El modelo analítico sugerido aquí muestra que la corrupción interactúa con la política siguiendo cuatro patrones o escenarios diferentes, y que estos informan las decisiones tomadas por líderes nacionales frente a las demandas de reforma. Como resultado, el modelo describe una dinámica que arroja luz sobre las razones detrás de los niveles actuales de implementación de iniciativas nacionales e internacionales contra la corrupción.

El documento está estructurado de la siguiente manera. La segunda sección revisa la literatura sobre reformas anticorrupción e identifica el dilema

\footnotetext{
Traducido al español por el autor.

Traducido al español por el autor.
} 
central que las afecta. En la tercera sección se presenta un modelo sistémico de corrupción inspirado en el trabajo de David Easton, describiendo los efectos que tiene la corrupción sobre la sociedad y el sistema político. La cuarta sección propone la existencia de cuatro escenarios de estrés sobre el sistema político causados por la presencia de corrupción, y da ejemplos ocurridos en el Perú en las últimas dos décadas. Finalmente, la quinta sección resume las conclusiones de este estudio.

\section{EL PROBLEMA CON LAS REFORMAS ANTICORRUPCIÓN}

\subsection{Revisión literaria}

Tradicionalmente hablando, la literatura sobre la lucha contra la corrupción ha estado dominada por un enfoque normativo basado en el modelo agente-principal (Kaufmann, 1998; Klitgaard, 1988; Pope, 1999). En forma muy breve, este modelo ve la corrupción como una consecuencia de las limitadas acciones e información disponibles para que los líderes puedan controlar el comportamiento de los funcionarios públicos, lo que resulta en abusos a la confianza pública. Ya sea que la figura de los «líderes» esté manifestada en la élite política (Doig, 1995; Khan, 2006; Man, 2009), la sociedad civil (Brunetti y Weder, 2003; Kisubi, 1999) o las organizaciones internacionales (Carr, 2006; Marong, 2002; Martin, 1999), el modelo se centra inevitablemente en las mejores estrategias disponibles para reducir la corrupción mediante la adopción y aplicación de políticas públicas y otras intervenciones sociopolíticas. Sin importar la utilidad relativa de las herramientas y estrategias desarrolladas en el marco de este modelo, empero, la persistencia de la corrupción en los países en desarrollo ha puesto en evidencia la importancia de la política por encima de las cuestiones técnicas.

Por lo tanto, al abordar la naturaleza intrínsecamente política de las iniciativas anticorrupción que habitualmente son impulsadas en países en desarrollo, un segundo grupo de estudios (denominado cleanups, o enfoque de la purgación) analiza la aparición de campañas anticorrupción en los países afectados endémicamente por este mal en los siguientes términos: «El impulso para limpiar la corrupción puede ser provisto principalmente por la exigencia política en lugar de por un genuino interés en el funcionamiento eficiente de las instituciones políticas y económicas de la nación» ${ }^{6}$ (Mbaku, 1996).

6 Traducido al español por el autor. 
Desde esta perspectiva, los esfuerzos contra la corrupción no se diseñan siguiendo consideraciones técnicas, sino más bien siguiendo los beneficios esperados que pueden producir en términos de capital político y concentración de poder; por lo tanto, tienden a ser muy temporales, limitados por la duración en el poder del gobierno que los adoptó (Kupatadze, 2012; Mbaku, 1996; Taylor, 2006).

Subrayando aún más la resistencia política a las reformas anticorrupción de corte técnico, es posible incluso ver la adopción de medidas de contrarreforma (desde la promulgación de reglamentos hasta reformas constitucionales) que facilitan la práctica de malversación pública: la censura de los medios de comunicación (Peters, 2003), la intervención del poder judicial y/o las agencias reguladoras (McMillan y Zoido, 2004; Sherman, 1980), la concentración de poder en el Ejecutivo (Corrales y Penfold, 2007), la multiplicación de regímenes especiales de contratación y adquisiciones (Schultz y Søreide, 2008), y la desactivación de canales formales para el monitoreo del gasto público (Roberts, 2000), entre otros.

Es siguiendo la línea de la literatura arriba presentada, que recientemente un nuevo enfoque, llamado Pensando y Trabajando Políticamente (Thinking and Working Politically, TWP), se ha solidificado en respuesta a problemas y fracasos comunes en la implementación de políticas públicas, apuntando abiertamente al papel crucial desempeñado por actores y contextos políticos locales y la necesidad de tenerlos claramente incluidos en cada etapa de análisis y ejecución. En el caso de los esfuerzos contra la corrupción, la lógica de este enfoque se puede encontrar en el trabajo de Hassid y Brass (2015), quienes discuten la diferencia entre China y Kenia en cuanto a su manejo de casos de corrupción, haciendo referencia a los niveles de presión y peligro experimentado por los Gobiernos de esos países. Bueno de Mesquita y Smith discuten un punto similar: «Si el individuo perjudicado por el cambio es el gobernante o el $\mathrm{CEO}$ - la misma persona que tiene que iniciar los cambios en primer lugarentonces podemos estar seguros que el cambio nunca va a suceder ${ }^{7}$ (2011, p. 252). Pero ya en los años ochenta el argumento estaba siendo ofrecido por Chubb y Vannicelli (1988), quienes, al discutir la forma en que los escándalos son manejados en Italia, se dieron cuenta efectivamente que «la paradoja se encuentra en el hecho de que la iniciativa para cualquier reforma de las instituciones públicas y cualquier moralización de la vida pública debe venir precisamente de aquellos actores políticos que están entre las causas principales de 
la degeneración $»^{8}$ (1988, p. 145). Por lo tanto, el enfoque TWP se basa en este razonamiento para atender a los temas relacionados a la corrupción, en contraste con la mayoría de los análisis tradicionales que consideraban (y que en cierta forma lo siguen haciendo, independientemente de sus argumentos formales) el trabajo anticorrupción como una cuestión inherentemente técnica.

\subsection{Argumento}

A partir de la revisión anterior, se hace evidente que los intereses particulares de los dirigentes políticos se encuentran en el centro de la discusión sobre la reforma anticorrupción, siendo incluso posible argumentar que reconocer la presencia de líderes tolerantes a la corrupción es aceptar la posible existencia de estrategias gubernamentales que pretenden defender (e incluso reforzar) un statu quo corrupto, independientemente de cualesquiera que sean las acciones tomadas por los impulsores internacionales y locales de la reforma propuesta. ¿Cómo podrían encajar en tal situación los verdaderos esfuerzos anticorrupción, entonces?

Sin hacer suposición alguna acerca de la naturaleza honesta o corrupta de los líderes políticos, la afirmación anterior es realista y significativa. Es realista en base a lo que se sabe copiosamente sobre los persistentes niveles de corrupción de alto nivel en la mayoría de países en desarrollo alrededor del mundo, y sobre el bajo nivel de adopción e implementación de las convenciones y declaraciones internacionales contra la corrupción. Podemos llamar a los Gobiernos de esos países apáticos, tolerantes o incluso corruptos; lo que importa es que reconozcamos la realidad de la falta de incentivos que enfrentan al considerar acciones de prevención y control: las políticas anti-corrupción tienen un costo, y el capital político no siempre (o incluso usualmente) es suficiente para renunciar a beneficios ilícitos.

La afirmación también es significativa porque abre la puerta para analizar las actividades gubernamentales no solo en términos de lo que se hace para implementar recomendaciones, sino también para impedirlas. La clave para empezar a explorar las consecuencias de este razonamiento será, entonces, adoptar explícitamente una descripción de la naturaleza de los actores gubernamentales en relación a su interés en controlar la corrupción.

Dado que las actividades gubernamentales nunca son gratuitas, la simple idea de llevar a cabo una acción destinada a combatir la corrupción nos obliga a considerar los costos inherentes a esa acción frente a sus posibles beneficios.

8 Traducido al español por el autor. 
Es esta relación beneficio-costo la que realmente refleja el valor de los esfuerzos anticorrupción para cualquier administración dada. Como el reducir la corrupción impacta positivamente en la sociedad, naturalmente genera beneficios para el Gobierno en forma de capital político, un tipo crucial de recursos que describe la cantidad de apoyo recibido de grupos internacionales y sociales en respuesta a circunstancias específicas. Con este apoyo, las autoridades pueden ejercer control y permanecer en el poder sin depender únicamente de la coerción. Por otra parte, la mayoría de las iniciativas contra la corrupción son dirigidas específicamente sobre actores gubernamentales (incluidos los líderes nacionales), creando así un costo adicional más allá de los regulares relacionados con la formulación y aplicación de políticas públicas. Si esos actores gubernamentales también están involucrados en actos de malversación pública, los beneficios ilícitos que perciban (además de su posición de élite) se verán amenazados y menguados en respuesta a sus propias acciones de gobierno. Como consecuencia, se puede esperar que una reforma anticorrupción significativa se estanque cuando los propios líderes están comprometidos en actos de corrupción, haciendo que la relación beneficio-costo sea una que favorezca la protección del statu quo.

Si bien este argumento no es claramente innovador, su exposición explícita rara vez ha sido utilizada como base en la construcción de modelos analíticos para explicar el fracaso de los esfuerzos anticorrupción. Por lo tanto, vale la pena comenzar este esfuerzo reconociendo primero que: para un gobierno honesto, las políticas anticorrupción serán atractivas solo en relación directa con el capital político que pueden generar; mientras que para un gobierno corrupto, las políticas anticorrupción serán evitadas en relación directa con los intereses que amenazan.

La discusión se mueve ahora a la construcción de un modelo que responda a esta premisa, el cual será acompañado en cada paso por casos empíricos que ilustren la validez del argumento en el devenir histórico de esfuerzos anticorrupción en Perú durante las últimas dos décadas.

\section{UN MODELO SISTÉMICO DE CORRUPCIÓN}

Para comenzar a elucidar las complejidades de la reforma anticorrupción, se requiere un conjunto inicial de conceptos que nos permitan discutir sobre los actores internacionales y nacionales, sus interacciones potenciales, y las condiciones y decisiones que los actores políticos podrían enfrentar con respecto a la corrupción en el sistema político. Este estudio considera al «modelo de 
respuesta dinámica del sistema político» desarrollado por David Easton (1965a, 1965b) como un buen lugar para comenzar, por lo que lo empleamos aquí para desentrańar la dinámica especial de la reforma anticorrupción.

El sistema político, en su descripción más básica, funciona gracias a la conversión de inputs (insumos) en outputs (productos). Los inputs son las demandas y apoyo recibidos por los actores gubernamentales; con estos, el sistema es capaz de producir outputs en forma de decisiones ejecutables dirigidas a afectar tanto a la sociedad como a su propio funcionamiento interno. Estos «intercambios» o "transacciones» (Easton 1965a, p. 109) entre el Gobierno y los actores de su entorno representan la vida del sistema político, la forma en que un país es manejado. Si bien las demandas son las materias primas utilizadas por el Gobierno para informar sus acciones, sin apoyo el Gobierno es incapaz de mantenerse en el poder y tener sus decisiones implementadas y ejecutadas, por lo que el flujo constante de apoyo al Gobierno es esencial para que el liderazgo ejerza autoridad. El apoyo, en estos términos, es indistinguible del capital político.

Las demandas tienden a afectar negativamente al Gobierno solo en relación a la forma en que disminuyen el nivel de apoyo cuando son desatendidas. Cuando las demandas aumentan, esto suele reflejar una situación insatisfactoria para la sociedad. En este sentido, la corrupción pública puede ser considerada como una fuente de estrés para el Gobierno debido al aumento de demandas que produce; y, debido a que la corrupción pública es realmente el estado de degeneración de las acciones de los actores gubernamentales en sus roles oficiales, también es un output. De hecho, el propio Easton identificó al clientelismo político (una forma de corrupción) como un tipo de output que genera estrés para el sistema mismo: «El recurrir a este tipo de outputs como fuente de apoyo específico para los objetos políticos puede resultar más eficaz en estresar que en mantener un sistema» (1965b, p. 361).

Empleando la terminología desarrollada en el trabajo de Easton, entonces, definiríamos la corrupción como un tipo de output que estresa al sistema, dando lugar a un aumento de las demandas y a una disminución del apoyo; si tal situación no es controlada, puede eventualmente conllevar no solo la caída de las autoridades, sino la insostenibilidad del propio sistema.

Por lo tanto, los titulares del Gobierno tienen un incentivo inherente para controlar el nivel de demandas mediante la adopción de medidas simbólicas y/o genuinas para aumentar el flujo de apoyo. Sin embargo, la forma específica

9 Traducido al espańol por el autor. 
que estas medidas puedan adoptar dependerá de los intereses secundarios de los responsables de la toma de decisiones políticas: ¿Es el control del poder político su objetivo final, o sus intereses están vinculados al statu quo? Tal es la diferencia entre una administración que apoya la reforma anticorrupción, y una que censura medios de comunicación y hace uso de populismo económico como forma de sobrevivir a una crisis de corrupción. Si bien ambas administraciones podrán reducir el estrés y estabilizar el nivel de apoyo político con igual efectividad, solo una de ellas resultará en nuevos estándares anticorrupción en el sistema.

Las consecuencias de definir la corrupción en estos términos son profundas y múltiples. Dando una lectura minuciosa de tal conceptualización a la luz del progreso actual de los estudios de corrupción, podemos argumentar que la corrupción produce estrés para el Gobierno siguiendo cuatro patrones o escenarios diferentes (que también determinarán las opciones disponibles para los líderes): ineficiencia en procesos, percepción pública, intolerancia adquirida y negligencia acumulada.

\section{Cuatro escenarios de CORRupción}

\subsection{Ineficiencia en procesos}

El primer escenario refleja la presencia de corrupción mediante sus efectos perjudiciales sobre el nivel de vida de los ciudadanos. Las condiciones resultantes suelen pertenecer a la esfera económica, pero también pueden adoptar otras formas. A pesar de que el nivel de corrupción en este escenario puede no ser percibido, ni estimado, directamente por el entorno social y/o internacional, una vez que las condiciones socioeconómicas se ven dańadas, el entorno reacciona retirando su apoyo a las autoridades y aumentando sus demandas. Sin embargo, como este escenario contempla únicamente las consecuencias indirectas de la corrupción (como la disminución de la eficiencia en los sistemas públicos o la calidad de la prestación de servicios), las demandas públicas no apuntan a la implementación y aplicación de medidas de control, sino a resolver problemas inmediatos (que son solo síntomas de corrupción).

Es posible reconocer tal escenario, y las estrategias gubernamentales adoptadas para reducir demandas y estimular el apoyo popular, en las reformas administrativas introducidas por el presidente Alberto Fujimori en Perú en los primeros años de la década de 1990.

La transferencia de poder entre los gobiernos del presidente Alan García Pérez (1985-1990) y el presidente Alberto Fujimori (1990-2000) tuvo lugar en medio 
de una profunda crisis económica, con un PBI per cápita contrayéndose en $-14 \%$ en 1989 y $-7 \%$ en 1990 , y una inflación disparándose a niveles de 3,398\% y 7,481\% en los mismos ańos (World Bank, 2015). Gran parte del problema se encontraba en las catastróficas políticas financieras y económicas implementadas por el gobierno de García, pero otro aspecto de la crisis era la depredación partidista de estructuras administrativas que había alcanzado peligrosos niveles bajo el control del APRA. Un diagnóstico realizado al poco tiempo de iniciarse la recuperación describió la situación en los siguientes términos:

El grave deterioro de la capacidad de gestión del Estado se manifiesta de múltiples maneras: creciente incapacidad para vigilar y supervisar el cumplimiento de las leyes, incapacidad para diseñar y ejecutar programas coherentes de política económica, vulnerabilidad y devaluación de la carrera administrativa, e incremento en la inmoralidad y corrupción en muchas instituciones del sector público (Cáceres y Paredes 1991, p. 143).

Para hacer frente a esta situación, la nueva administración adoptó medidas encaminadas a restablecer la economía peruana, lo que también implicó la implementación de acciones anticorrupción para el control de los ilícitos burocráticos. Tal enfoque era posible porque los intereses de la nueva administración no estaban ligados a la preservación de estructuras partidistas rapaces en los niveles inferiores del aparato estatal; en cambio, el gobierno de Fujimori buscó la centralización del poder político y de las redes de corrupción en las esferas más altas del Estado (Peña-Mancillas, 2011). Por lo tanto, en lugar de combatir la corrupción en todas sus formas, lo que el nuevo Gobierno hizo fue abordar la crisis económica a través de medidas de control específicas para mejorar la administración pública, reducir demandas, estimular el apoyo popular y generar recursos financieros suficientes para alimentar una nueva red de corrupción levantada alrededor del presidente Fujimori y su asesor Vladimiro Montesinos.

La microcorrupción en la recaudación de impuestos (Baca, 2000) fue abordada de inmediato, ya que esta fuente de ingresos proveía en 1990 solo el 7,9\% del PBI del país. La autoridad nacional de recaudación de impuestos de Perú, Sunat, y el Banco de la Nación fueron reformados estructuralmente, mientras que otras agencias conexas sufrieron simplemente el despido masivo de trabajadores que habían ingresado y engordado el aparato estatal durante los cinco años anteriores (Durand, 2005). Diferentes agencias reguladoras fueron rápidamente creadas, caracterizadas "por contar con un alto grado de autonomía de los procesos políticos, recursos humanos calificados, procesos de gestión basados en resultados y fuentes de financiamiento propias» (Straface y Basco, 2006, p. 10). Estas acciones solo fueron efectivas en la medida de lo necesario, y no se extenderían a otras organizaciones del Estado igualmente afectadas por altos niveles 
de corrupción, como el Poder Judicial, ya que estas no eran directamente responsables de la recuperación económica y podían en cambio amenazar los intereses de la nueva administración. Según Carol Wise, «el presidente no tenía otra opción que reformar las instituciones estatales que eran críticas para la recuperación económica ${ }^{10}$ (Santiso, 2004, p. 294), y al hacerlo redujo efectivamente la microcorrupción y estimuló el apoyo popular (Hunt, 2005).

La figura 1 ofrece una idea más clara, aunque a nivel micro, de la relación entre la recuperación económica y el apoyo popular durante los primeros años de la administración de Fujimori. Como es posible apreciar, una vez que el índice de precios de consumo (datos para la ciudad de Lima) se estabiliza a mediados de 1992, también lo hace el nivel de aprobación popular del presidente Fujimori. De hecho, St. John señala que «cada vez más peruanos aprobaron las políticas de Fujimori, especialmente la estabilización de la economía» ${ }^{11}$ (2017, p. 69), lo cual es empíricamente corroborado por Weyland (2000) y Arce (2002), quienes demuestran que el apoyo popular al presidente Fujimori estuvo estadísticamente relacionado a sus reformas económicas, en particular aquellas relativas al control de la inflación.

Figura 1. Índice de precios de consumo en Lima y aprobación popular del presidente, 1989-1994

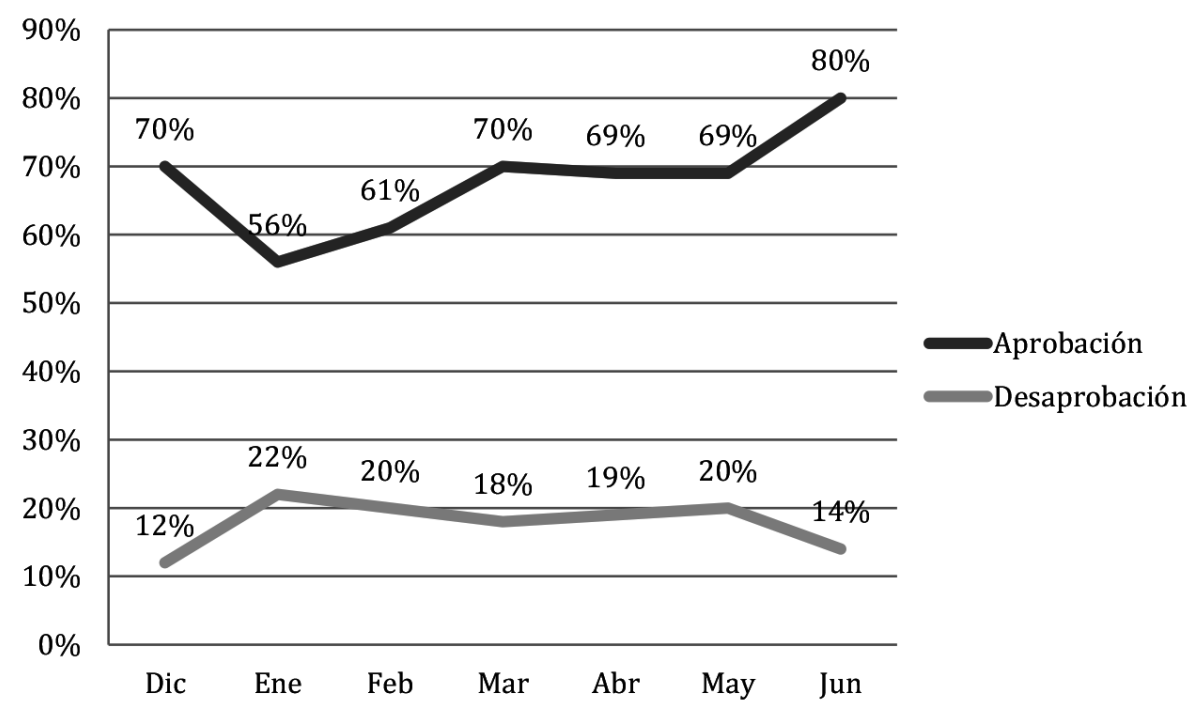

Fuente: Tanaka (1998) y Reyna (2000).

\footnotetext{
10 Traducido al espańol por el autor.

11 Traducido al espańol por el autor.
} 
En cuanto a la corrupción de alto nivel, en cambio, el Gobierno llegó al punto de cancelar un préstamo ya aprobado por US\$ 25 millones del Banco Mundial para la implementación de reformas en el Poder Judicial, porque no estaba dispuesto a aceptar ciertas condiciones con respecto a otorgarle mayor autonomía e independencia (Guerra García, 1999). Esto, por supuesto, sería solo el comienzo de un gran proceso de captura del Estado que transformó al Gobierno en una mafia.

\subsection{Percepción pública}

En el segundo escenario, el cual es también el más común, una actividad corrupta que involucra a uno o más miembros de la élite gobernante es percibida por actores domésticos (o internacionales) fuera de la esfera pública, creando lo que se conoce como un escándalo de corrupción. En este caso, el output (actividad ilícita) producido por las autoridades es incompatible con las expectativas de los ciudadanos y, por lo tanto, provoca la expresión de demandas para una efectiva lucha contra la corrupción y la reducción del apoyo al Gobierno, causando estrés al sistema.

Encontramos un ejemplo de este tipo de escenario durante el escándalo de Petrogate en 2008, el cual nos da una clara imagen de los niveles de estrés sobre el sistema que los escándalos de corrupción pueden generar, y las formas en que el Gobierno puede manejar estas situaciones.

El evento comenzó de forma explosiva el 5 de octubre de 2008, cuando cuatro grabaciones de audio (tomadas de manera subrepticia) fueron presentadas en un importante programa político de televisión. En ellos, Alberto Químper Herrera, director de la empresa peruana para la promoción de la inversión en el sector de hidrocarburos, Perupetro, podía ser escuchado discutiendo con el exministro del APRA Rómulo León Alegría el pago de sobornos para adjudicar contratos petroleros a Discover Petroleum, una empresa noruega (La República, 2008a). El contrato, de hecho, había sido adjudicado con éxito a Discover Petroleum el 10 de septiembre.

Frente a las explosivas revelaciones, la reacción del Gobierno fue inmediata: esa misma noche, César Gutiérrez, presidente de Petroperú (la empresa peruana de petróleos de propiedad del Estado), se vio obligado a presentar su renuncia; el ministro de Energía y Minas Juan Valdivia Romero siguió el ejemplo; y el presidente Alan García pidió enérgicamente la detención inmediata de Alberto Químper, a quien denominó «rata» frente a los medios de comunicación.

Al día siguiente se adoptaron combinaciones de respuestas simbólicas y genuinas, con el presidente García aceptando la renuncia de Valdivia y expresando: 
«Hasta que tu honorabilidad, como lo quieres, quede debidamente comprobada, te acepto la renuncia dejando en claro que eres un hombre honesto, pero te acepto la renuncia» (Andina, 2008a). Con fuerza igual pero opuesta, el partido APRA procedió a expulsar a Rómulo León de sus filas, declarando: «El APRA lamenta profundamente que haya personas inescrupulosas que se aprovechen delincuencialmente del sacrificio de miles de militantes» (La República 2008b). Por último, la administración prosiguió a suspender los contratos adjudicados a Discover Petroleum, e incluso ordenó que se retirase la bandera de Noruega que ondeaba frente al edificio de Petroperú (Caretas, 2008a), en un signo claramente destinado a mostrar su resolución frente al escándalo.

Sin embargo, nuevas grabaciones de audio siguieron, planteando preguntas con respecto a la cantidad de información que el Gobierno había tenido realmente sobre los negociados antes de que la historia estallase en las noticias. La cobertura mediática y el descontento popular aumentaron, y en el Congreso se prepararon mociones de interpelación e incluso de censura contra miembros del Gobierno.

En estas circunstancias, el presidente García no tuvo otra opción que hacer concesiones políticas significativas y aceptar la renuncia formal del Consejo de Ministros en su totalidad (Chirinos, 2008), ofreciendo el cargo de primer ministro al líder independiente Yehude Simon Murano, quien disfrutaba ya de cierta popularidad por su honestidad y su compromiso con la transparencia en el sector público. En relación a esta decisión, la revista política Caretas expresó:

¿Qué significa la elección de Simon por parte de Alan García? Primero, la importante reducción en el peso del aparato aprista, que retiene solamente las carteras de Jorge Villasante en Trabajo y Enrique Cornejo en Vivienda. Drástico ajuste de cuentas con la bancada oficialista que, en Jorge del Castillo, dejó caer sin más a uno de los suyos (ver entrevista siguiente). Con el anterior premier el partido ostentaba un nivel orgánico de influencia que hoy ya no existe (2008b, p. 11).

Además, la decisión representó una declaración clara (aunque simbólica) sobre la posición de la administración en materia de corrupción, como también reconoció Caretas: «La lucha contra la corrupción, tal como se lo planteó el presidente García, ocupará buena parte de la agenda [de Simon]» (2008b, p. 13). Esta muestra de voluntad política para la reforma anticorrupción, junto con el cambio de seis de sus ministros (el resto fueron ratificados individualmente en sus cargos), demostró ser eficaz para aumentar la aprobación popular del Ejecutivo. Según una encuesta realizada entre el 22 y el 24 de octubre, su nivel de aprobación popular se había mantenido estable en $16 \%$, mientras que la del presidente García había aumentado del 19\% en septiembre al 22\%; 
el apoyo al ex primer ministro Del Castillo también subió un 4\%, a pesar de que el $84 \%$ de los encuestados era de la opinión que él había estado al tanto del ilícito previo al destape de los audios (Apoyo, 2008a). En opinión de Alfredo Torres, director de Apoyo:

[e]l impacto del nombramiento [de Simon] sobre la imagen presidencial ha sido moderado... pero hay que tener en cuenta que, si no se hubiese producido el cambio de gabinete, la aprobación a la gestión presidencial habría sufrido probablemente una caída significativa como consecuencia del escándalo de los "petroaudios» (Apoyo 2008a, p. 1).

Sin embargo, los medios de comunicación y los partidos de oposición mantuvieron el escándalo en la agenda pública. El evento se vio especialmente impulsado por el hecho de que León seguía como fugitivo de la justicia y que los exministros Del Castillo, Garrido Lecca y Juan Valdivia estaban siendo investigados formalmente por su posible participación y responsabilidad en el ilícito. Las constantes denuncias contra actores cercanos al presidente García hicieron que la aprobación de este y la del Poder Ejecutivo cayeran 3\% y 1\% a mediados de noviembre, respectivamente, con un $42 \%$ de encuestados expresando desaprobación debido al alto grado de corrupción que afectaba al Gobierno (frente a un $37 \%$ que había sido de esa opinión el mes anterior). El ambiente doméstico expresó escepticismo sobre el discurso de García en relación a la lucha contra la corrupción: el 56\% creía que la administración no había hecho nada para abordar esta cuestión desde que había asumido el cargo en 2006, y el 38\% consideraba que la recompensa de S/. 100 mil (aproximadamente US \$ 30 mil) ofrecidos para la captura de León el 5 de noviembre era una mera cortina de humo desplegada por el Gobierno (Apoyo, 2008b).

En medio de esta presión continua (reflejada, y en parte representada, por las noticias de corrupción aparecidas en la portada del diario La República a lo largo del $78 \%$ de este tiempo —-mucho mayor al promedio de $24 \%$ para otros periodos-); sin embargo, la situación fue finalmente resuelta gracias a la adopción de dos medidas separadas, y al inicio de un nuevo evento de alto nivel.

Primero, el 13 de noviembre, Rómulo León se entregó a las autoridades y fue inmediatamente arrestado; con ello, un alto grado de cobertura sensacionalista desapareció rápidamente, pasando a otros temas. Cabe señalar, sin embargo, que ninguna condena sería impuesta como consecuencia de este caso, ya que todos los implicados fueron finalmente liberados y todos los cargos retirados, lo cual brinda motivos para considerar la decisión de León como un arreglo político para convertirse en un chivo expiatorio estratégico.

Segundo, pocas semanas luego de que el primer ministro Yehude Simon asumiese el cargo, fue presentado a debate público un Plan Nacional Anticorrupción 
basado en el trabajo ya adelantado por el Gobierno anterior, en 2006. La iniciativa ayudó a estimular el apoyo político hacia Yehude Simon y al presidente García, con el primero subiendo $2 \%$ entre noviembre y diciembre, mientras que la desaprobación a la administración de García debido a la corrupción se redujo al 34\% (Apoyo, 2008c). Más allá de su papel político, sin embargo, está claro que el Plan no pretendía ser mucho más que una medida simbólica: A pesar de haber sido presentado en el prominente foro del Acuerdo Nacional (Andina, 2008b) y haber recibido comentarios positivos por parte de la sociedad civil organizada (Proética, 2008), nunca sería adoptado oficialmente por el Gobierno, por lo que se puede decir que su impacto en los estándares anticorrupción también resultó simbólico en el mejor de los casos.

Tercero, a partir del 18 de noviembre los medios de comunicación pasaron a la cobertura de la Cumbre del APEC celebrada en Lima, que llenó la mayoría de las portadas hasta el día 24. El manejo público de la cumbre ayudó al presidente García y al Poder Ejecutivo a recuperarse rápidamente de los costos del escándalo de Petrogate, subiendo de vuelta al 25\% y 21\% de aprobación popular a mediados de diciembre, respectivamente. Cuando se le preguntó por las razones de su apoyo político a la administración de García, el 38\% de los encuestados mencionó su desempeño durante la reunión de APEC (Apoyo, 2008c). Así, para todo propósito práctico, la cumbre de APEC tuvo el mismo efecto que una cortina de humo, beneficiando al Gobierno al empujar el escándalo de Petrogate fuera de la agenda pública.

\subsection{Intolerancia adquirida}

El tercer escenario no describe una situación en la que la corrupción es indirectamente sufrida ni directamente percibida, sino que refleja un cambio en los paradigmas en los que la corrupción es abordada por el entorno nacional e internacional antes de que se produzcan nuevos escándalos. Este es el caso de la aparición del movimiento internacional anticorrupción, y de la transformación general del entorno hacia reglas y procedimientos más estrictos para prevenir y disuadir la participación de actores públicos en actos ilícitos. Aunque la evolución de este movimiento es gradual y tiene momentos de intensidad altos y bajos, en general se puede decir que el entorno experimenta una disminución evidente en los niveles de corrupción que está dispuesto a tolerar, y como resultado intenta efectuar la implementación de nuevas políticas públicas contra la corrupción en el contexto nacional a través de diferentes formas de influencia y presión. Esta influencia y presión es lo que hemos estado denominando demandas. Cuando las autoridades, a su vez, no cumplen con estas exigencias 
a través de actividades anticorrupción apropiadas, el apoyo disminuye y se dice que el sistema está bajo estrés.

La evolución constante del movimiento internacional anticorrupción, y la presión que ello significa para la estabilidad del statu quo peruano debido a la consiguiente reducción en tolerancia, se evidenció en la forma en que la administración del presidente Ollanta Humala (2011-2016) manejó sus compromisos con la Alianza para el Gobierno Abierto (OGP, por sus siglas en inglés), una "plataforma intergubernamental para reformistas nacionales comprometidos en hacer que sus gobiernos sean más abiertos, responsables y sensibles a los ciudadanos» ${ }^{12}$ (OGP, 2015).

La intención de ingresar a la OGP reflejó en un principio la reconocida posibilidad de ganar reputación internacional: La Carta de Intención, después de todo, fue enviada el 15 de septiembre de 2011, menos de dos meses después del inicio del nuevo gobierno, y solo cinco días antes que la OGP fuese lanzada oficialmente junto con la celebración de la 66a Asamblea General de las Naciones Unidas. Así, poco después de que se comunicasen los requisitos para ingresar a la Alianza, el país inició un proceso de coordinación entre diferentes organismos públicos y organizaciones civiles para el desarrollo de un Plan de Acción en consenso, que debía estar listo en abril de 2012.

El proceso de redacción y aprobación del Plan, a pesar de poder ser considerado formalmente exitoso, expuso una serie de cuestiones relativas a la distribución de facultades y funciones entre los principales organismos anticorrupción del país, especialmente la Secretaría de Gestión Pública (SGP) y la Comisión de Alto Nivel Anticorrupción (CAN), ambas parte de la Presidencia del Consejo de Ministros (PCM). Un miembro de la SGP recuerda:

Al principio la cancillería convocó a la sociedad civil y a la Defensoría del Pueblo a una reunión para introducir el tema, pero no invitaron a la SGP. Más tarde, obviamente, se le dijo a la Cancillería que la SGP debía estar presente ya que es la oficina responsable en materia de transparencia y vigilancia ciudadana, y así es como se nos invita recién desde la segunda reunión y se crea el grupo de trabajo. ${ }^{13}$

A modo de contraste, y a pesar de su rol formal, la CAN es relegada y excluida:

La CAN entró en escena más tarde. Al principio, los miembros del Comité Ejecutivo eran sólo la Defensoría del Pueblo, la Contraloría General de la República, el Ministerio de Relaciones Exteriores y la SGP; estos eran los cuatro representantes del aparato estatal. Y de parte de la sociedad civil estaban Proética, Ciudadanos al Día, el Consejo

2 Traducido al español por el autor.

13 Entrevista personal. Lima, 23 de octubre, 2014. 
de Prensa Peruana y la Asociación Nacional de Centros. La inclusión de la CAN se llevó a cabo más adelante... Una vez que el proyecto fue redactado, mantuvimos reuniones con otras agencias: primero, hubo reuniones con todos los sectores, y luego otras reuniones donde también se incluyeron a las diferentes oficinas de la PCM. Fue en ese momento cuando la CAN fue finalmente informada de lo que se estaba haciendo, y cuando comienza a tener una participación más activa. ${ }^{14}$

De esta forma, el proceso no solo afectó las relaciones entre ambas oficinas, sino que también puso de relieve la intención de despolitizar el significado de gobierno abierto vis a vis la administración pública y la formulación de políticas. Un alto funcionario de la CAN explicó:

Susana Silva, Coordinadora General de la CAN, fue nombrada el 19 de noviembre de 2011, y la primera reunión del Gobierno Abierto fue entre el 22 y el 25 de noviembre. La SGP, la Defensoría del Pueblo y la CAN fueron convocadas, pero Silva no pudo ir porque estaba en el proceso de asumir cargo... Así que más tarde, cuando trató de unirse, la SGP le dijo: «No, esto es un asunto exclusivamente de transparencia; no está conectado con la lucha contra la corrupción». Así que nos echaron... ${ }^{15}$

La animosidad entre estas oficinas fue agravada aún más cuando en enero de 2013 el Gobierno decidió poner a la SGP (que tenía escasos recursos humanos y ya había transferido la mayor parte de sus tareas de vigilancia a la CAN) a cargo de la comisión permanente multisectorial para el seguimiento del Plan, mientras que la CAN (que acababa de recibir rango de Ley como el principal foro contra la corrupción en el país) fue dejada completamente fuera ${ }^{16}$.

De hecho, el primer informe del Mecanismo Independiente de Monitoreo (IRM) cita repetidamente la falta de coordinación y cooperación como fuente de debilidad, tanto para la CAN como para la realización de las actividades contenidas en el Plan de Acción, sugiriendo la responsabilidad del bajo nivel de implementación a la falta de voluntad política del Gobierno central:

[Existen] preocupaciones recurrentes entre los especialistas consultados: Que el Plan de Acción solo contará con apoyo político en la medida en que no amenace el statu quo existente. El investigador [del IRM] considera esta creencia como válida mientras el Gobierno no cree una entidad con la suficiente autonomía, autoridad y jurisdicción para alinear a todo el Estado dentro de un estándar de gobierno abierto (Casas, 2014, p. 44).

La conclusión del IRM resultaría ser una evaluación dolorosamente precisa durante el proceso de elaboración de un nuevo Plan de Acción para el periodo 2014-2015, que incluyó esta vez la creación de una Autoridad Autónoma de

\footnotetext{
14 Entrevista personal. Lima, 23 de octubre, 2014.

15 Entrevista personal. Lima, 29 de octubre, 2014.

16 Es necesario indicar que la CAN sería eventualmente incorporada.
} 
Transparencia (AAT), propuesta por la Defensoría del Pueblo y la sociedad civil. Frente a esta demanda a comprometerse a crear una entidad con las características sugeridas por el IRM, el Gobierno optó por renegar el proceso y rechazó aprobar el proyecto de documento. En octubre de 2014, más de cuatro meses después de que el plan propuesto había sido acabado, la ONG Proética (capítulo peruano de Transparencia Internacional) comenzó a instar públicamente al gobierno de Humala a respetar sus compromisos con el proceso de la OGP (Proética, 2014a). El 27 de noviembre, el Comité Directivo de la OGP adoptó una posición similar y envió una comunicación oficial al Secretario General de la PCM:

Países con más de cuatro meses de retraso en la presentación de su Plan Nacional de Acción (NAP) serán considerados como actuando en contra del proceso de la OGP [...] Por lo tanto, esta carta es para informarle que el Gobierno del Perú ha actuado en contra del proceso de la OGP para este ciclo de desarrollo del plan de acción [...] (Proética, 2014b).

Cuando el Gobierno finalmente presentó su propia versión del documento, el cual abiertamente omitía cualquier referencia a la creación de una AAT, el plazo objetivo del plan original tuvo que ser revisado, conformándose así con ser aplicable para el periodo 2015-2016, dejando al 2014 como un ańo perdido para el gobierno abierto en Perú. Así, el manejo de la participación de Perú en la OGP evidenció el peso relativo del capital político frente a la estabilidad del statu quo para los intereses de la administración de Humala, siendo que el Gobierno estuvo dispuesto a sacrificar cierto nivel de apoyo con tal de evadir cualquier reforma anticorrupción significativa.

Mientras que varias voces independientes dentro y fuera de la CAN apoyaron la creación de una ATT, el Gobierno no estaba dispuesto a ver tal proyecto convertido en realidad. El primer obstáculo para la AAT había venido, naturalmente, de la SGP. El mismo alto funcionario de la CAN declaró:

No creo que haya problemas constitucionales [detrás de la AAT]... Estoy 100\% de acuerdo con [la creación de] una AAT. Hemos estado tratando de empujarla hacia adelante por un largo tiempo, pero bueno, las cosas no están avanzando como querríamos. La SGP, de hecho, entregó un informe técnico diciendo que la AAT no era viable, que [la propuesta] era un documento mal producido. Así que se produjo todo un problema porque la Defensoría del Pueblo había enviado el proyecto legislativo a la PCM en el marco de una reunión de la CAN, y el Secretario General nos la había remitido a nosotros y a la SGP... [Luego de eso, la CAN] había comenzado a coordinar, a trabajar, a celebrar varias reuniones con [la SGP], y [un día] de repente nos informaron que la SGP ya había elaborado un informe negativo... El tema fue discutido al más alto nivel, y dijeron que iba a ser reformulado. Hubo mucha presión de parte de la sociedad civil porque sabían que había una posición negativa de la SGP, por lo que 
estos últimos retrocedieron y se ofrecieron a elaborar un nuevo proyecto legislativo... La nueva propuesta es casi la misma, excepto que establece que la AAT no será una agencia autónoma, y que no quitará funciones a la SGP17.

Es sintomático que, cuando el defensor del pueblo propuso por primera vez la creación de una AAT en abril de 2012, la vicepresidenta Marisol Espinoza había expresado el compromiso de la administración de Humala de apoyar ese esfuerzo (Andina, 2012). Sin embargo, según el analista político Carlos Basombrío (Castillo, 2015), a finales de 2014 el progreso de las fuerzas contrareformistas y la debilidad del gobierno de Humala en términos políticos apuntaba a un estancamiento completo de las actividades estructurales contra la corrupción, lo cual fue precisamente evidenciado en la exclusión de la AAT del segundo Plan de Acción de la OGP.

\subsection{Negligencia acumulada}

Por último, los patrones de corrupción en términos sistémicos pueden reducir aún más los niveles de apoyo a través de la exposición prolongada del sistema al estrés en cualquiera de los escenarios anteriores. En otras palabras, este escenario refleja las consecuencias de dejar (por negligencia o incapacidad) que la ineficiencia en los procesos, la percepción pública y/o la intolerancia adquirida a la corrupción sofoquen el flujo de apoyo hacia el Gobierno hasta un punto de virtual colapso. En estas condiciones, el nivel de estrés ejercido sobre el sistema afecta no solo las posibilidades de que un determinado conjunto de autoridades permanezca en el poder, sino que erosiona el apoyo público a todo el régimen político, a sus instituciones y a la estructura jurídica del país.

El fin del gobierno de Fujimori (1990-2000) y las medidas tomadas por el gobierno de transición del presidente Valentín Paniagua (2000-2001) describen este escenario.

Después de años de presión creciente por parte de grupos de oposición respecto a la creciente centralización del poder en manos del presidente y de su círculo cercano, el gobierno de Fujimori finalmente alcanzó un punto de quiebre en setiembre del año 2000. Mientras el gobierno seguía lidiando con niveles considerables de presión nacional por causa de su victoria en unas elecciones presidenciales marcadas por acusaciones de fraude y abuso de poder, y habiendo apenas sobrevivido a un escándalo internacional que involucraba al asesor presidencial Vladimiro Montesinos en la venta ilegal de armas a las FARC de Colombia, el mayor escándalo habría de explotar aún en la noche

17 Entrevista personal. Lima, 29 de octubre, 2014. 
del 14 de setiembre. En conferencia de prensa, miembros de la oposición en el Congreso presentaron un video que mostraba a Montesinos, sentado en una pequeńa oficina del Sistema de Inteligencia Nacional, negociando con el congresista Alberto Kouri su renuncia al partido opositor Perú Posible y su incorporación a las filas del partido de gobierno.

Este fue el golpe final que expuso de una vez por todas la verdadera naturaleza y el papel del asesor presidencial, que durante una década había estado construyendo una red de corrupción sobre el aparato estatal y el sector privado. Las noticias de alta corrupción gubernamental llenaron las portadas del diario La República casi todos los días durante más de dos meses, mientras que representantes de la Iglesia católica del Perú, el defensor del pueblo, las asociaciones del sector privado, los líderes de la oposición e incluso el Gobierno de los Estados Unidos exigían la pronta investigación, captura y procesamiento de Vladimiro Montesinos (La República, 2000a). Los congresistas de oposición adoptaron la misma postura y amenazaron con boicotear las sesiones del Congreso a menos que una comisión de investigación fuese debidamente conformada (Chirito, 2000). La tasa de desaprobación del presidente Fujimori (que había aumentado desde las elecciones) se disparó, alcanzando el 77,7\% (La República, 2000b).

Las noticias sobre las actividades ilícitas de Montesinos aumentaron rápidamente, estimuladas por el descubrimiento de millonarias cuentas bancarias asociadas a él en un número de países (Teran Vega, 2000; La República, 2000c), que presuntamente habían sido resultado de negociados con traficantes de drogas peruanos y colombianos, malversación de fondos y un gran número de actividades delictivas. Blanca Nélida Colán, exagente de Montesinos en el Ministerio Público, fue reemplazada en su cargo de fiscal de la Nación, así como lo fue José Portillo Campbell como jefe de la ONPE. El vicepresidente Francisco Tudela renunció a su puesto el 23 de octubre y la presidenta del Congreso Martha Hildebrandt fue despojada de su cargo el 13 de noviembre.

La presión del entorno estaba llegando a un punto en el que la ejecución normal de las actividades gubernamentales estaba siendo amenazada. La administración había quedado casi sin estrategias para hacer frente a la presión de la sociedad civil, la oposición política y los actores internacionales. El 13 de noviembre, el procurador público José Ugaz y su equipo presentaron una solicitud formal de investigación contra el presidente Alberto Fujimori; ese mismo día, sin embargo, Fujimori huía con dirección a Brunei (y de allí a Japón).

Fujimori se aferró a la preservación del statu quo mientras, a la par, producía constantes outputs de corrupción e ignoraba acciones cruciales de control, 
acumulando niveles progresivos de presión de todos los actores externos, hasta que la situación se volvió inmanejable. Al final, el sistema político no solo fue confrontado con la crisis del statu quo, sino que el estrés se había extendido al resto de la vida política de la nación. Controlado por las fuerzas políticas en el poder, el Congreso había dejado de operar y el monopolio de la fuerza física estaba siendo activamente disputado. El sistema no podía continuar sin emprender una verdadera reforma anticorrupción.

En estas circunstancias, las medidas adoptadas por el Gobierno de transición que tomó las riendas no solo fueron positivas para la democracia peruana, sino también inevitables. El colapso del gobierno de Fujimori le dio la oportunidad a la oposición política de hacerse con el control del gobierno y comenzar a implementar las reformas necesarias para hacer frente al estrés, pero para ello la adopción y aplicación de reformas anticorrupción reales (y otras medidas democráticas cruciales) debían ser garantizadas. El funcionamiento del sistema político dependía en ese momento de la satisfacción efectiva de las demandas anticorrupción.

La importancia de la reforma anticorrupción como piedra angular de la agenda de la administración, y no solo como forma de depuración política, se reflejó claramente en la creación de la Iniciativa Nacional contra la Corrupción (INA), un foro oficial para el debate y propuesta de políticas anticorrupción que incluyó actores de las esferas pública y privada y de la sociedad civil. La tarea encomendada a la INA fue descrita por el ministro de Justicia García-Sayán:

Es indispensable avanzar en los lineamientos de un plan nacional contra la corrupción. No es un plan de gobierno transitorio, lo cual excedería ampliamente su mandato, sino elaborar lineamientos que puedan ser asumidos por el Estado y la sociedad peruana... Este proyecto global será entregado a las autoridades que asuman la conducción del país el próximo 28 de julio (Zileri, 2001).

El valor de ir más allá de medidas simbólicas y/o paliativas durante esos cruciales días fue declarado explícitamente por un alto funcionario del gobierno de transición:

El tema de la moralidad pública y la corrupción iba más allá de una situación temporal, sin desmedro de lo grave que era, y lo vimos y discutimos mucho con Paniagua como un asunto estructural... Teníamos que ver una forma de, además de adoptar esas medidas para afrontar la corrupción que teníamos delante, definir políticas preventivas contra la corrupción que involucren políticas públicas, el diseño de estrategias institucionales, la inclusión de la sociedad civil, campañas de sensibilización y educación, etc. ${ }^{18}$.

18 Entrevista personal. Lima, 7 de noviembre, 2014. 
Así, bajo el gobierno de transición de Paniagua, los estándares anticorrupción nacionales sufrieron un cambio dramático. Este periodo vio la satisfacción de las demandas anticorrupción, tanto en términos de ejecución como de reforma, con casi ningún output de corrupción estresando adicionalmente al sistema. El Gobierno se encargó de abordar el escenario de negligencia acumulada e invirtió una cantidad importante de recursos logísticos y políticos en reformar el sistema sin recurrir a medidas puramente simbólicas para generar apoyo. El resultado fue el retorno a una sana democracia en el Perú, y el exitoso desarrollo de nuevas elecciones presidenciales y congresales bajo una administración que llegó a contar con el apoyo del $80 \%$ del país, algo que ningún otro presidente peruano ha podido igualar hasta hoy. La figura 2 muestra la evolución de su aprobación popular, que refleja la aplicación de medidas anticorrupción y la normalización de las demandas anticorrupción.

Figura 2. Aprobación presidencial de Valentín Paniagua

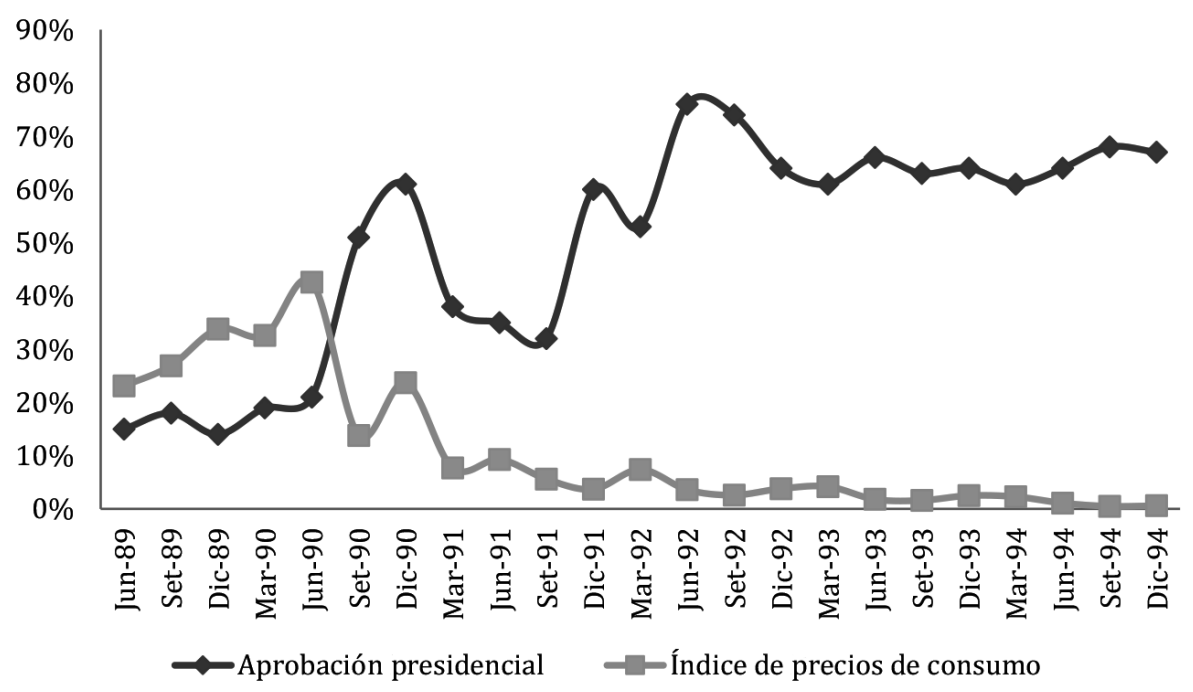

Fuente: Apoyo (2001).

Con la normalización de la vida política, sin embargo, se puso fin al escenario de negligencia acumulada, al igual que a la lucha anticorrupción del Gobierno central. Un miembro de la INA comentaría más tarde: «Es posible afirmar que ninguno de los gobiernos [de los presidentes Toledo y García] implementó las sugerencias de la INA». ${ }^{19}$ Una anécdota contada por un alto funcionario

19 Entrevista personal. Lima, 21 de octubre, 2013 
del gobierno de Toledo nos ayuda a vislumbrar el nivel de voluntad política para acciones de lucha contra la corrupción que existió luego que se hubiese asegurado suficiente apoyo para el sistema:

Casi nadie en el Ministerio (de Justicia) conocía [del documento Un Perú Sin Corrupción] que había sido publicado por la INA. ¿Cómo era eso posible, si había sido entregado? La edición impresa salió sesenta o noventa días después de la toma de posesión del presidente Toledo... Entonces, ¿`ómo fue posible? ¿Dónde estaban los folletos? ¿No sabían los funcionarios públicos [de su existencia]? ... Se dieron órdenes para investigar y localizar los documentos. Para mayor sorpresa [del ministro de Justicia], fueron encontrados en cajas almacenadas en un baño fuera de servicio. Nunca se les había distribuido ${ }^{20}$.

\section{Conclusiones}

La discusión realizada en las páginas anteriores sobre las condiciones y patrones de acción anticorrupción revelan la compleja dinámica y los desafíos que enfrentan los actores nacionales e internacionales involucrados en la lucha contra la corrupción pública. El hecho de que este fenómeno ilícito deba ser entendido como produciendo diferentes tipos de estrés para el Gobierno, y que el significado de ese estrés solo pueda ser interpretado considerando los intereses particulares de los líderes políticos, nos obliga a apartarnos del análisis común y unidimensional de medidas de prevención y control. Los escenarios explorados aquí desentrañan así las condiciones bajo las cuales el Gobierno se ve forzado a responder, ya sea adoptando estrategias de defensa para proteger al statu quo, o cediendo ante demandas anticorrupción. Pero también nos permiten estudiar y diseñar estrategias anticorrupción desde diferentes perspectivas, dependiendo del escenario en que deseemos enfocarnos, con sus efectos y dinámicas particulares.

El argumento presentado aquí obliga a la literatura sobre reformas anticorrupción a reconocer explícitamente y aceptar la existencia de incentivos para mantener el statu quo anticorrupción, y a construir futuros modelos teóricos basados en esa realidad. Al pasar por alto los incentivos reales que diferentes grupos de autoridades tienen para obstaculizar reformas, los esfuerzos pasados para formular recomendaciones significativas han producido escasos resultados, y por lo tanto han causado que el escepticismo se extienda. Mientras que en ciertos casos anecdóticos esas recomendaciones pueden haber obtenido resultados positivos, no hay una lógica científica que defienda el seguir financiando intervenciones basadas en supuestos a priori sobre la voluntad política de líderes nacionales.

20 Entrevista personal. Lima, 21 de octubre, 2013. 
Para los líderes políticos en sociedades corruptas, la función pública representa no solo poder político, sino también una forma de beneficiarse económicamente. En esos lugares, tanto el capital político como los beneficios corruptos deben ser considerados parte de la estructura de incentivos para la vida política, por lo que el actual movimiento internacional contra la corrupción pierde su tiempo y dinero apelando solo al primer elemento, sin tener en cuenta el segundo. Es por eso que la implementación de políticas anticorrupción ha sido continuamente decepcionante durante los últimos veinte años.

$\mathrm{Al}$ abordar las limitaciones inherentes a la reforma anticorrupción, el presente estudio brinda la oportunidad de emplear los recursos del movimiento internacional contra la corrupción de una manera más eficaz y eficiente, mediante una evaluación realista de contextos sociales y políticos. Si estas consideraciones pueden ser incorporadas en futuras estrategias de investigación y campañas prorreforma, entonces este campo de estudio podría ver en el futuro un nivel de éxito que el pasado hasta ahora le ha negado.

\section{REFERENCIAS}

Apoyo (2001). Informe de Opinión: Lima, junio del 2001. http://www2.congreso.gob.pe/sicr/ cendocbib/cat_uibd.nsf/primero/19BFD7E1ED2D6751052574FF007B5273?opendocument

Apoyo (2008a). Esperanza en Yehude. Opinión Data, 8(105). https://www.ipsos.com/sites/default/ files/publication/2009-11/Opinion_Data_Octubre_2008.pdf

Apoyo (2008b). Conflictos, corrupción y APEC. Opinión Data 8(106). http://www.ipsos.pe/sites/ default/files/opinion_data/Opinion_Data_Noviembre_2008.pdf.

Apoyo (2008c). Aprobación presidencial y Plan Anticrisis. Opinión Data, 8(107). https://www. ipsos.com/sites/default/files/publication/2009-11/Opinion_Data_Diciembre_2008.pdf

Arce, M. (2002). Violencia política y aprobación presidencial en el Perú. Debates en Sociología, (27), $175-187$.

Baca, J. (2000). El ancla fiscal: la reforma tributaria. En R. Abusada, F. Du Bois, E. Morón y J. Valderrama (eds.), La reforma incompleta. Rescatando los noventa (163-212). Lima: Centro de Investigación de la Universidad del Pacífico; Instituto Peruano de Economía.

Brunetti, A. y Weder, B. (2003). A Free Press is Bad News for Corruption. Journal of Public Economics, (87), 1801-1824. https://doi.org/10.1016/S0047-2727(01)00186-4

Bueno de Mesquita, B. y Smith, A. (2011). The Dictator's Handbook: Why Bad Behavior is Almost Always Good Politics. Nueva York: PublicAffairs.

Cáceres, A. y Paredes, C. (1991). Diagnóstico de la situación económica a fines de 1989. En C. Paredes y J. Sachs (eds.), Estabilización y crecimiento en el Perú (pp. 132-154). Lima: Grade.

Carr, I. (2006). Fighting Corruption Through Regional and International Conventions: A Satisfactory Solution? (Legal Series, Working Paper 1864).

Casas, J. (2014). Mecanismo de Revisión Independiente: El Perú-Informe de avance 2012-13. Recuperado del Open Government Partnership. http://www.opengovpartnership.org/sites/default/ files/Peru-Final-2012-Web.pdf. 
Chubb, J. y Vannicelli, M. (1988). Italy: A Web of Scandals in a Flawed Democracy. En A. S. Markovits y M. Silverstein (eds.), The Politics of Scandal: Power and Process in Liberal Democracies (pp. 122-150). Nueva York: Holmes \& Meir Publishers.

Corrales, J. y Penfold, M. (2007). Venezuela: Crowding Out the Oppositio. Journal of Democracy, 18(2), 99-113. https://doi.org/10.1353/jod.2007.0020

Doig, A. (1995). Good Government and Sustainable Anti-Corruption Strategies: A Role for Independent Anti-Corruption Agencies? Public Administration and Development, 15(2), 151-165. https://doi.org/10.1002/pad.4230150206

Durand, F. (2005). Dinámica política de la corrupción y participación empresarial. En F. Portocarrero (Ed.), El pacto infame: estudios sobre la corrupción en el Perú (pp. 287-330). Lima: Universidad del Pacífico.

Easton, D. (1965a). A Framework for Political Analysis. Englewood Cliffs, NJ: Prentice-Hall.

Easton, D. (1965b). A Systems Analysis of Political Life. Chicago, IL: The University of Chicago Press.

Guerra García, G. (1999). La reforma del Estado en el Perú. Pautas para reestructurar el Poder Ejecutivo. Lima: Agenda.

GTCC (2010). Informe Anual sobre la Lucha contra la Corrupción en el Perú. http://www.oas.org/ juridico/pdfs/mesicic4_per_gtcc.pdf

Hassid, J. y Brass, J. N. (2015). Scandals, Media and Good Governance in China and Kenya. Journal of Asian and African Studies, 50(3), 325-342. https://doi.org/10.1177/0021909614528865

Hunt, J. (2005). Why Are Some Public Officials More Corrupt Than Others? William Davidson Institute, Working Paper 790.

Kaufmann, D. (1998). Challenges in the Next Stage of Anti-corruption. En D. Kaufmann (ed.), New Perspectives on Combating Corruption (pp. 139-163). Washington, DC: Economic Development Institute.

Khan, M. H. (2006). Governance and Anti-Corruption Reforms in Developing Countries: Policies, Evidence and Ways Forward. G-24 (Discussion Papers Series No. 42). Nueva York; Ginebra: United Nations Conference on Trade and Development.

Kisubi, M. M. (1999). Involving Civil Society in the Fight against Corruption. En R. Stapenhurst y S. J. Kpundeh (eds.), Curbing Corruption: Towards a Model of Building National Integrity (pp. 117-125). Washington DC: The World Bank.

Klitgaard, R. (1988). Controlling Corruption. Berkeley, CA: University of California Press.

Kupatadze, A. (2012). Explaining Georgia’s Anti-Corruption Drive. European Security, 21(1), 16-36. https://doi.org/10.1080/09662839.2012.656597

Man, M. (2009). Political Corruption in Russia: An Evaluation of Russia's Anti-Corruption Strategies, 1991-2009. POLIS Journal, (2), 1-53.

Marong, A. (2002). Toward a Normative Consensus Against Corruption: Legal Effects of the Principles to Combat Corruption in Africa. Denver Journal of International Law and Policy, (30), 99-129.

Martin, A. T. (1999). The Development of International Bribery Law. Natural Resources \& Environment, 14(2), 95-102.

Mbaku, J. M. (1996). Bureaucratic Corruption in Africa: The Futility of Cleanups. Cato Journal, 16(1), 99-118.

McMillan, J. y Zoido, P. (2004). How to Subvert Democracy: Montesinos in Peru. (CESifo Working Paper, No. 1173). https://doi.org/10.1257/0895330042632690

OECD (2014). Open Government in Latin America. OECD Public Governance Reviews. París: OECD Publishing.

OECD (2017). OECD Integrity Review of Peru: Enhancing Public Sector Integrity for Inclusive Growth. OECD Public Governance Reviews. París: OECD Publishing. 
OGP (2015). Open Government Partnership. http://www.opengovpartnership.org.

Peña-Mancillas, V. S. (2011). Combatir la corrupción en el Perú: a diez años de Fujimori. Revista del CLAD Reforma y Democracia, (51), 211-240.

Peters, B. (2003). The Media's Role: Covering or Covering Up Corruption? (Global Corruption Report). Recuperado de http://unpan1.un.org/intradoc/groups/public/documents/apcity/ unpan008437.pdf

Persson, A., Rothstein, B. y Teorell, J. (2010). The Failure of Anti-Corruption Policies - A Theoretical Mischaracterization of the Problem (QoG Working Paper Series, 19). The Quality of Government Institute, University of Gothenburg.

Pope, J. (1999). Elements of a Successful Anticorruption Strategy. En R. Stapenhurst y S. J. Kpundeh (eds.), Curbing Corruption: Toward a Model for Building National Integrity (pp. 97-104). Washington, DC: World Bank.

Proética (24 de diciembre, 2008). Proética desestima críticas a Plan Anticorrupción y destaca compromiso del Ejecutivo en esa labor. Recuperado de http:/www.proetica.org.pe/noticias/ proetica-desestima-criticas-a-plan-anticorrupcion-y-destaca-compromiso-del-ejecutivo-en-esalabor/

Reyna, C. (2000). La Anunciación de Fujimori: Alan García 1985-1990. Lima: DESCO Centro de Estudios y Promoción del Desarrollo.

Roberts, A. S. (2000). Less Government, More Secrecy: Reinvention and the Weakening of Freedom of Information Law. Public Administration Review, 60(4), 308-320. https://doi.org/ 10.1111/0033-3352.00093

Santiso, C. (2004). Re-forming the state: governance institutions and the credibility of economic policymaking. International Public Management Journal, 7(2), 271-298.

Schultz, J. y Søreide, T. (2008). Corruption in Emergency Procurement. Disasters, 32(4), 516-536. https://doi.org/10.1111/j.1467-7717.2008.01053.x

Sherman, L. W. (1980). Three Models of Organizational Corruption in Agencies of Social Control. Social Problems, 27(4), 478-491. https://doi.org/10.2307/800176

St. John, R. B. (2017). Peruvian Foreign Policy in the New Millennium: Continuity and change. RIRA, 2(2), 65-119. https://doi.org/10.18800/revistaira.201702.002

Straface, F. y Basco, A. I. (2006). La reforma del Estado en Perú. BID, Working Paper. https:// publications.iadb.org/handle/11319/3783

Tanaka, M. (1998). Los Espejismos de la Democracia: El colapso de partidos en el Perú, 1980-1995, en perspectiva comparada. Lima: IEP Instituto de Estudios Peruanos.

Taylor, S. D. (2006). Divergent Politico-Legal Responses to Past Presidential Corruption in Zambia and Kenya: Catching the 'Big Fish', or letting him off the hook? Third World Quarterly, 27(2), 281-301. https://doi.org/10.1080/01436590500432325

UNDP (2012). Anticorruption Programmes in Latin America and the Caribbean. Study on Anti-Corruption Trends and UNDP Projects. United Nations Development Programme-Undp-Undp Regional Centre Panama. https://www.bcn.cl/obtienearchivo?id=documentos/10221.1/57225/3/Anticorruption_Programs.pdf

Weyland, K (2000). ¿La paradoja del éxito? Los determinantes del apoyo político al presidente Fujimori. Debates en Sociología, (25-26), 213-244.

World Bank (2015). World DataBank. http://databank.worldbank.org/data/home.aspx 


\section{Prensa}

Andina (2008a). Presidente García acepta la renuncia del ministro Valdivia (ampliación). 6 de octubre. Recuperado de http://www.andina.com.pe/agencia/noticia-presidente-garcia-aceptarenuncia-del-ministro-valdivia-ampliacion-197406.aspx

Andina (2008b). Jefe del Gabinete presenta hoy Plan Anticorrupción ante Acuerdo Nacional. 14 de noviembre. Recuperado de http://www.andina.com.pe/agencia/noticia-jefe-del-gabinetepresenta-hoy-plan-anticorrupcion-ante-acuerdo-nacional-203842.aspx

Andina (2012). Proponen crear autoridad nacional autónoma para transparencia y acceso a la información pública. 25 de abril. Recuperado de http://www. andina.com.pe/agencia/noticiaproponen-crear-autoridad-nacional-autonoma-para-transparencia-y-acceso-a-informacionpublica-409679.aspx

Caretas (2008a). Final del Faenón. Nro. 2048, 9 de octubre.

Caretas (2008b). Golpe de Simon. Nro. 2049, 16 de octubre, pp. 10-14 y 93.

Castillo, C. (2015). Carlos Basombrío: 'La corrupción ha ganado terreno en este gobierno'. Perú21, 4 de enero. Recuperado de https://peru21.pe/politica/carlos-basombrio-corrupcionganado-terreno-gobierno-160215

Chirinos, C. (2008). García acepta renuncia en pleno. BBC, 11 de octubre. Recuperado de http://news.bbc.co.uk/hi/spanish/latin_america/newsid_7664000/7664823.stm

Chirito, J. (2000). La oposición no regresará al Pleno. La República, 16 de setiembre, p. 6B.

La República (2000a). Ante un Perú indignado, Inexplicable silencio de Fujimori: Sin Habla. 16 de setiembre, p. 1.

La República (2000b). Rechazo nacional a Fujimori. 7 de octubre, p. 1.

La República (2000c). Montesinos se levantó mil millones. 6 de noviembre, p. 1.

La República (2008a). Cuatro audios sacuden Palacio. 6 de octubre, p. 2.

La República (2008b). APRA expulsa a perpetuidad a León. 7 de octubre, p. 4.

Teran Vega, C. (2000). Montesinos tiene US $\$ 48$ millones en cuentas secretas de tres bancos suizos. La República, 3 de noviembre, p. 2.

Zileri, M. (2001). Operación Tenaza. Caretas, Nro. 1666. 